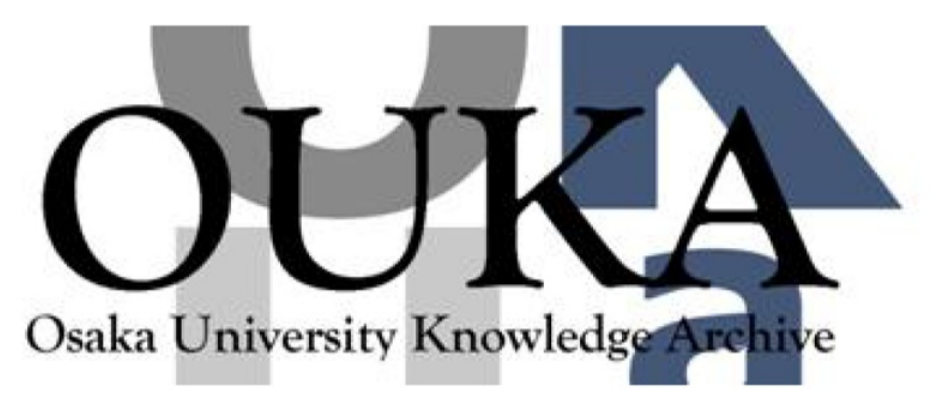

\begin{tabular}{|c|l|}
\hline Title & $\begin{array}{l}\text { Birefringent encoding and multichannel } \\
\text { reflective correlator for optical array logic }\end{array}$ \\
\hline Author(s) & Tanida, Jun; Nakagawa, Jun; Ichioka, Yoshiki \\
\hline Citation & Applied Optics. 27(18) p. 3819-p.3823 \\
\hline Issue Date & $1988-09-15$ \\
\hline oaire:version & VoR \\
\hline URL & https://hdl. handle.net/11094/2950 \\
\hline rights & \\
\hline Note & \\
\hline
\end{tabular}

Osaka University Knowledge Archive : OUKA

https://ir. Library. osaka-u. ac. jp/

Osaka University 


\title{
Birefringent encoding and multichannel reflective correlator for optical array logic
}

\author{
Jun Tanida, Jun Nakagawa, and Yoshiki Ichioka
}

\begin{abstract}
A simple image encoding method and a flexible correlator for optical array logic are presented. The encoding method uses the birefringence phenomenon and produces spatial code patterns in parallel for all pixels in two input images. The flexible correlator is composed of a multichannel illuminating system and a segmented reflector, which executes flexible correlation for optical array logic. Using the proposed encoding method together with this correlator, an efficient optical array logic processor capable of executing various parallel neighborhood operations can be constructed.
\end{abstract}

\section{Introduction}

Using the physical characteristics of light, largecapacity high-speed information processing can be achieved, which cannot be implemented by conventional electronic techniques. Light propagation in free space offers attractive advantages, such as parallelism and crosstalk-free interconnection, so that optical techniques using these features give a promise of massively parallel information processing. Optical 2D Fourier transformation, ${ }^{1}$ symbolic substitution, ${ }^{2}$ and optical perfect shuffle interconnection ${ }^{3}$ are such examples.

We proposed optical array logic (OAL) as a generalpurpose optical parallel processing technique and showed its processing procedures for practical uses. ${ }^{4,5}$ $\mathrm{OAL}$ is a technique to construct any parallel neighborhood operation for two 2-D binary data. Parallel neighborhood operations can be designed, described, and executed systematically. OAL has a close relationship to both array $\operatorname{logic}^{6}$ and cellular $\operatorname{logic}^{7}$ in electronics, so that their programming resources can be used in OAL. In addition, using the concept of pattern logic ${ }^{5}$ various kinds of parallel processing are possible with OAL.

OAL is implemented by three procedures, i.e., encoding of images to be processed, correlation for the coded image, and on operation for the correlated im-

The authors are with Osaka University, Department of Applied Physics, Suita, Osaka 565, Japan.

Received 17 December 1987.

0003-6935/88/183819-05\$02.00/0.

(c) 1988 Optical Society of America. ages. These procedures can be executed in parallel with optical techniques, and then parallel operations are achieved. However, the encoding procedure has been difficult to implement, and development of an efficient coding method holds the key for practical use of OAL.

In this paper we propose a simple encoding method using the phenomenon of birefringence ${ }^{8}$ : we call it birefringent encoding. Then we present a specially designed correlator for efficient implementation of OAL; we call that a multichannel reflective correlator.

We show a birefringent encoding method in Sec. II, and present a multichannel reflective correlator for OAL in Sec. III. Using the proposed encoding method together with the multichannel reflective correlator, a simple OAL processor is constructed. Experimental results by the OAL processor are demonstrated in Sec. IV. We discuss the expandability of the proposed encoding method and correlator in Sec. V.

\section{Birefringent Encoding}

OAL is a technique to achieve any parallel neighborhood operation for two 2-D binary data according to the procedures shown in Fig. 1.4,5 Two 2-D binary data points are encoded into a coded image in which every pixel is converted into any one of four spatial code patterns according to a combination of values of corresponding pixels in the input data. The coded image is separately correlated with several pointwise functions called operation kernels. The individual correlated images are spatially sampled at 1-pixel intervals along the vertical and horizontal directions. Inverted oR operation for all the sampled images provides the result of a parallel neighborhood operation, whose data format is changed to match that of the inputs in the next step. Since the processing manner 


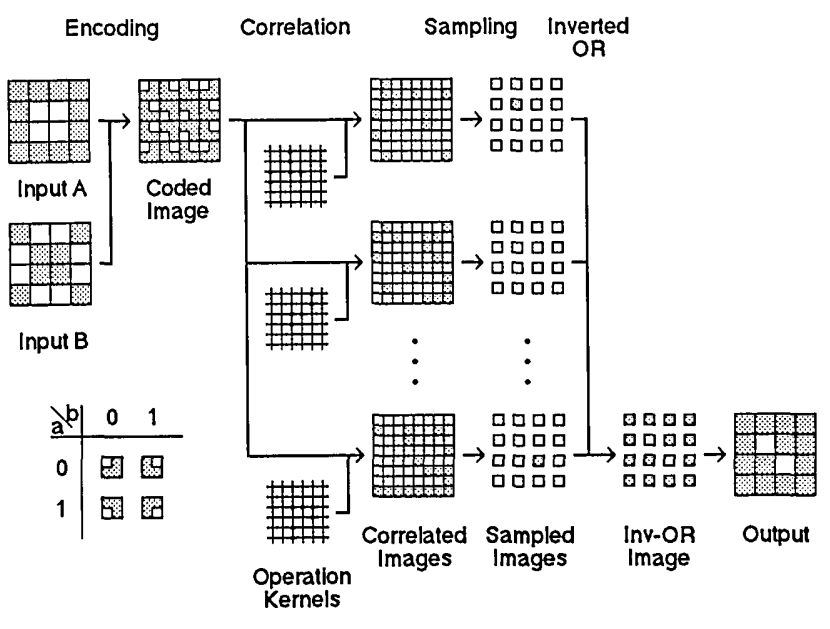

Fig. 1. Schematic diagram of optical array logic.

in OAL is identical to the sum of product processing, any logical operation for 2-D data can be achieved by a combination of operation kernels.

Encoding is an important procedure in OAL because it occurs in every processing cycle. Thus development of an efficient encoding method holds the key to the practical use of OAL. The encoding process is identical for all pixels in the image, so that the use of optoelectronic hybrid devices can be considered in a practical implementation. ${ }^{9}$ Although the electronic encoding method may be a practical solution, we present here an optical encoding method that is simpler and more reliable than the electronic one.

The new method uses the phenomenon of birefringence in a uniaxial crystal, ${ }^{8}$ which converts polarizing information into spatial data. The basic principle of the method is the same as that of a digital light deflector ${ }^{10}$ and polarization logic by Lohmann et al..$^{11}$ except for parallelism and space variance. In this proposed method the digital light deflector is used to encode a set of corresponding pixels in the two input images. Operating the defelctors for individual sets of pixels in parallel, we can obtain a coded image from two input images at a time.

Figure 2 shows the principle of birefringent encoding. For simplicity, the coding process of corresponding pixels, $a_{i j}$ and $b_{i j}$, is depicted. A horizontally polarized light beam (called the probe beam) passes through the controllable halfwave plate $1(C H P 1)$, the birefringent crystal 1 ( $B C 1$ ), $C H 2$, and $B C 2$. The plane containing the $z$ axis and the optical axis $(O A)$ of $B C 1$ is set perpendicular to that containing the optical axis of $B C 2$. Operating modes of $C H P 1$ and CHP2 are controlled according to values of $a_{i j}$ and $b_{i j}$, respectively, that is, if $a_{i j}$ is zero, the polarizing direction of the light passed through $C H P 1$ is rotated at $90^{\circ}$, and if $a_{i j}$ is one, the polarizing direction is preserved. The operating mode of CHP2 is controlled dependent on the value of $b_{i j}$ in the same manner as that of CHP 1 .

As shown in Fig. 2, horizontally polarized light passes through $B C 1$ as the ordinary ray, whereas vertically polarized light is the extraordinary ray. The

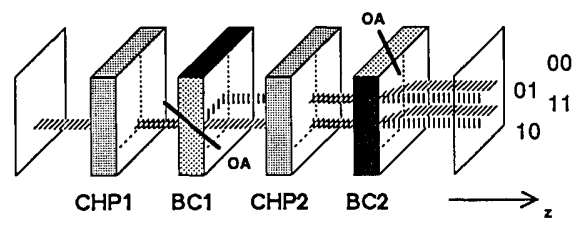

Fig. 2. Principle of birefringent encoding, $O A$ indicates the optical axis.

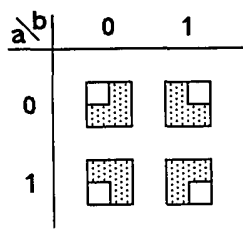

(a)

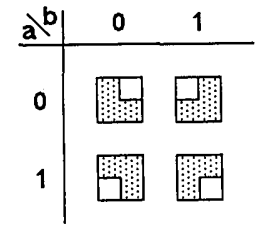

(b)
Fig. 3. Two sets of code patterns: (a) original OAL and (b) obtained by birefringent encoding.

\begin{tabular}{|c|c|c|c|c|c|c|c|}
\hline Function & symbol & $\begin{array}{l}\text { Krnl Unt } \\
\text { of Orig. }\end{array}$ & $\begin{array}{l}\text { Krnl Unt } \\
\text { of B.C. }\end{array}$ & Function & Symbol & $\begin{array}{l}\text { Krni Unt } \\
\text { of Orig. }\end{array}$ & $\begin{array}{l}\text { Krnl Unt } \\
\text { of B.C. }\end{array}$ \\
\hline 1 & .. & \# & \# & $a+b$ & PP & \# & \# \\
\hline$\overline{0}+\bar{b}$ & NN & \# & \# & $a \bullet b$ & uv & \# & \# \\
\hline $\overrightarrow{0}+b$ & NP & \# & \# & $b$ & .1 & \# & \# \\
\hline $\bar{a}$ & 0. & \# & \# & $\bar{a} \mathrm{~b}$ & 01 & \# & \# \\
\hline$a+\bar{D}$ & PN & \# & \# & a & 1. & \# & \# \\
\hline $\overrightarrow{\mathrm{D}}$ & .0 & $\#$ & $\#$ & $a \bar{b}$ & 10 & \# & \# \\
\hline$\overline{a \bullet b}$ & EE & \# & \# & 00 & 11 & $\#$ & \# \\
\hline$\overline{\mathbf{a}} \overrightarrow{\mathrm{b}}$ & 00 & \# & \# & 0 & on & $\#$ & $\#$ \\
\hline
\end{tabular}

Fig. 4. Kernel units for original OAL and that using birefringent encoding.

ordinary ray passes straight through a birefringent crystal such as calcite, but the extraordinary ray is diverted. Thus two different optical passes are produced depending on the value of $a_{i j}$. The identical process is carried out by combined operation of $C H P 2$ and $B C 2$. As a result, four different passes are made. Thus, in an observation plane behind $B C 2$, we can obtain a light spot at any one of four different positions ignoring the state of polarization. The position of the light spot depends on a combination of the $a_{i j}$ and $b_{i j}$ values. Note that, although the encoding output is a light spot in the above explanatory case, square code patterns as shown in Fig. 1 can also be obtained using a light bundle whose cross section is a quarter pixel square size as the probe beam.

The relationship between the input data $a_{i j}$ and $b_{i j}$ and the resultant code patterns is slightly different from that of the original OAL as shown in Fig. 3. This is caused by the inherent nature of this method and cannot be avoided; however, this difference is not an essential problem. Using the kernel units (Krnl Unit of B.C.) in Fig. 4 instead of the original ones (Krnl Unit of Orig.), this problem can be easily solved. Here, a kernel unit is a functional primitive in an operation kernel, which determines the nature of the operation to be executed for the corresponding pixels in the inputs. 
Birefringent encoding has many advantages such as simplicity, stability, parallel nature, and light power efficiency. A spatial light modulator plays the role of a controllable halfwave plate. The thickness and the cutting direction of the birefringent crystal determines the split distance of the extraordinary ray. Thus the thinner the thickness of the birefringent crystal, the greater the number of processing points. The proposed encoding method is quite stable unlike the holographic method. ${ }^{12}$

\section{Multichannel Reflective Correlator}

Correlation as well as encoding is an important operation in OAL. Although many optical correlation methods and correlators have been suggested, ${ }^{13}$ a specially designed correlator is required for efficient implementation of OAL. The reason is that one operand of the correlation in OAL is a pointwise function (operation kernel). So that the necessary correlation is regarded as an operation in which the coded image is shifted with various offsets and the shifted images are overlapped to be the result. The shadow-casting14 and multifocus imaging systems ${ }^{15}$ are good examples of the correlator for OAL.

In OAL the offset value of shifting and the combination of overlapping are defined graphically with the dots in the operation kernel. A dot located at $m$ row and $n$ column corresponds to an image shifted horizontally by $-m d / 2$ and vertically by $-n d / 2$, where $d$ is the pixel size (or double the size of the structural cell of the coded image). A combination of the dots indicates overlapping of shifted images.

The performance of the correlator for OAL is determined by the allowable shifting distance and the number of overlapping images. If a neighborhood operation for large neighborhood area is attempted, a large size operation kernel is required. The size of the operation kernel is determined by the allowable shifting distance and the number of overlapping images. In other words, the performance indices of the correlator directly correspond to the size of an operation kernel and the total number of dots in the operation kernel.

However, the neighborhood area is not always square; it depends on the sort of operation to be executed. Furthermore, the number of dots in an operation kernel is smaller than that of possible points in the operation kernel, and frequently the dots localize as discussed in Ref. 5. Therefore, a correlation system capable of flexibility composing operation kernels is desirable for efficient implementation of OAL.

We consider a new type of correlator that can virtually synthesize various sizes of operation kernel, using multichannel illumination and a segmented reflector. Figure 5 shows one version of the multichannel reflective correlator. The collimated light beam coming from a point source in the point source array illuminates an input object and produces the image of the source on the corresponding mirror in the segmented mirror. After reflection, the image of the input is focused on the output plane where the image is shifted according to the tilt angle of the mirror. Every set of

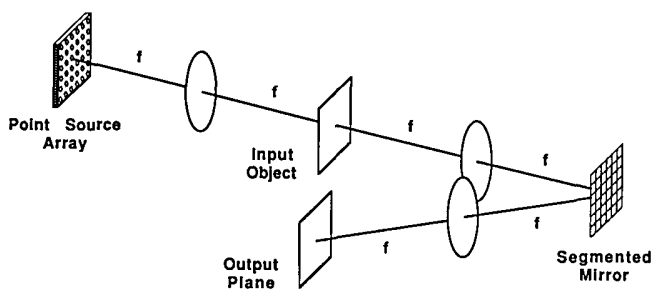

Fig. 5. Optical system of multichannel reflective correlator.

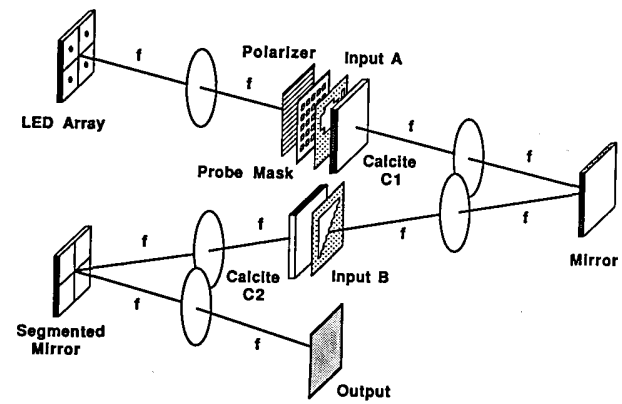

Fig. 6. Experimental system composed of a birefringent encoder and a multichannel reflective correlator.

point source and mirror forms one illuminating channel, which independently provides a shifted image of the input. Thus, controlling the switching configuration of point sources and tilt angle of the mirrors, we can implement parallel correlation with large operation kernels.

The advantages of the multichannel reflective correlator are as follows. First, the correlator can flexibly synthesize any operation kernel with any size. As long as the total number of dots in an operation kernel is less than the channel number of the correlator, correlation with any operation kernel is possible by the correlator. Second, the correlator has high efficiency for light-power transmission. Since it is composed of reflective elements, it can operate without power loss. Third, the correlator can easily be constructed. It consists of conventional optical components and we need no special devices, such as a 2-D optical shutter, as required in the multifocus imaging system. ${ }^{15}$

\section{Experimental Verification}

The birefringent encoding method and correlation by means of the multi-channel reflective correlator are useful techniques to construct an efficient OAL processor. We constructed the actual optical system to verify the principles and usefulness of both techniques.

Figure 6 shows the experimental setup. Inputs $A$ and $B$ consist of square halfwave plates of $2 \mathrm{~mm}$ on each side, with square spaces of the same size. Figure 7 shows two input images in which shaded square cells mean halfwave plates and white cells are spaces. Thus pixels corresponding to white cells have values of one and those to shaded cells have values of zero. To fix the position of every pixel and to prevent overlapping of adjacent coded patterns, a probe mask is put on input $A$. 


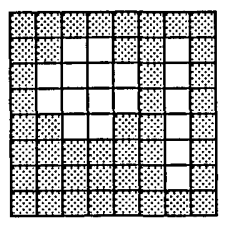

(a)

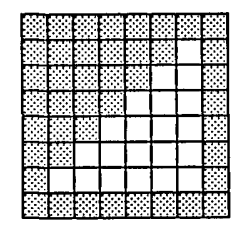

(b)
Fig. 7. Input data for operations: (a) input $A$ and (b) input $B$.

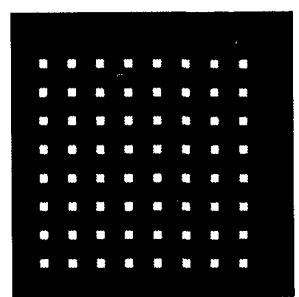

(a)

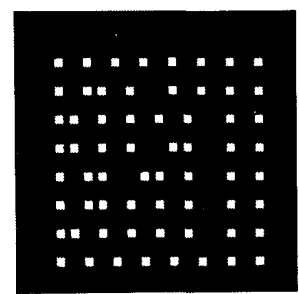

(c)

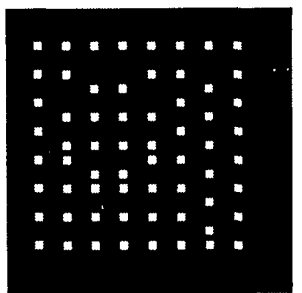

(b)

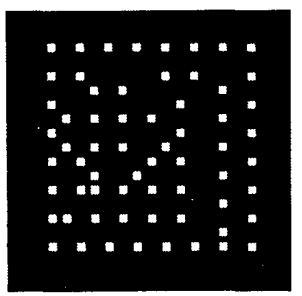

(d)
Fig. 8. Experimental results of birefringent encoding: (a) without calcite, (b) with calcite $C 1$, (c) with calcite $C 2$, and (d) with calcites $C 1$ and $C 2$.

Calcite cyrstals are used as birefringent crystals, to produce the $1-\mathrm{mm}$ shift for the extraordinary ray after passing through the crystal; $2 \times 2$ LEDs are used as a point source array. The segmented mirror is composed of $2 \times 2$ square mirrors which can be manually adjusted. Thus the constructed correlator has four channels.

Figure 8 shows the experimental results of the birefringent encoding. Figure 8(a) is the picture obtained with no calcite. Figure $8(\mathrm{~b})$ is the result when the calcite $C 1$ was set in the optical path. Figure 8(c) is that when the calcite $C 2$ was set in the optical path. Figure $8(\mathrm{~d})$ is that when both $C 1$ and $C 2$ were set in the optical path, which indicates the coded image. Figures 3 and 6 show that the desired code patterns have been obtained.

Various kinds of operation kernel are synthesized and correlated with the coded image produced by the birefringent encoder. Figure 9 shows the results of correlation using the experimental setup in Fig. 6 . Four kinds of operation kernel are synthesized and corresponding logical operations are shown in the left column. Observing the correlated images in the middle column through a sampling mask, we can obtain the results of desired logical operations in the right column. The results are obtained by dark-true logic in which the dark signal is assigned to logical one and the
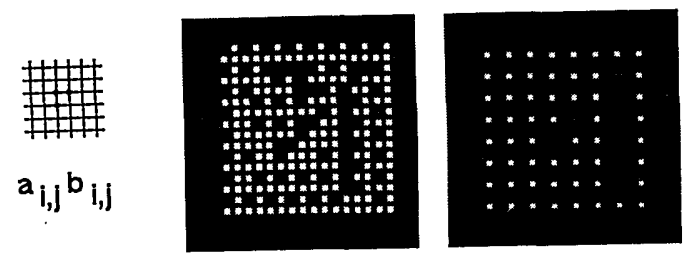

(a)
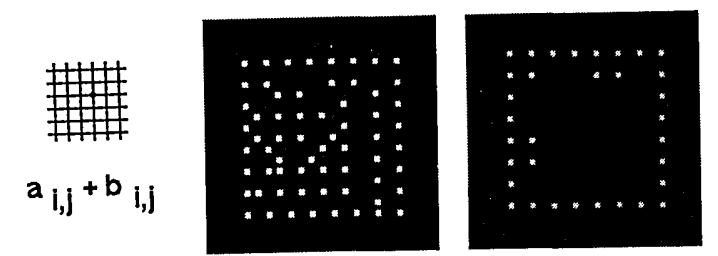

(b)

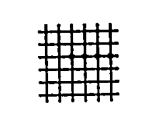

$a_{i, j} a_{i, j+1}$
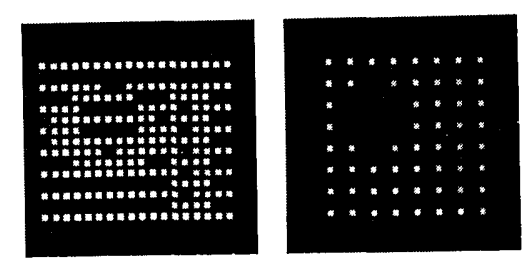

(c)
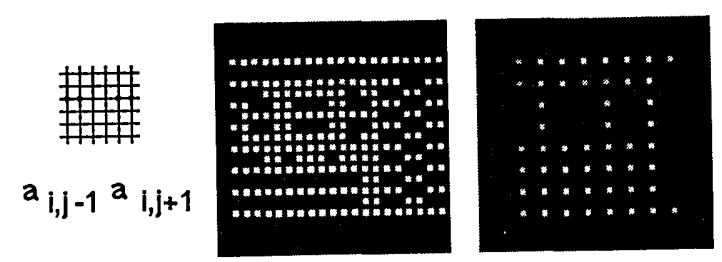

(d)

Fig. 9. Experimental results of correlation and logical operation: (a) AND, (b) OR, and (c), (d) differentiations. Synthesized operation kernels, equivalent operation, correlated image, and sampled image are shown for each result.

bright signal to logical zero. The sort of operation is determined by the operation kernel consisting of kernel units in Fig. 4. Although the experimental system has only four channels, various kinds of operation can be executed.

\section{Discussion}

The most important factor in evaluating the performance of parallel processing is the number of the data points to be processed: the greater the number of processing points in the system, the better the performance. In the birefringent encoding method, the separation distance between ordinary ray and extraordinary ray, which corresponds to half-pixel, can be determined by the thickness and the cutting direction of the birefringent crystal. This means that the birefringent encoding method does not limit the pixel size. Therefore, the number of data points to be processed is mainly restricted by the spatial resolution of the controllable halfwave plate.

Another restriction is the signal spread on the segmented mirror. Increase in the processing points causes overlapping of adjacent channels, i.e., crosstalk noise. For a fixed size optical system, the smaller the size of each mirror, the more the channel number. Thus optimal design is required to get full power out of the correlator. 


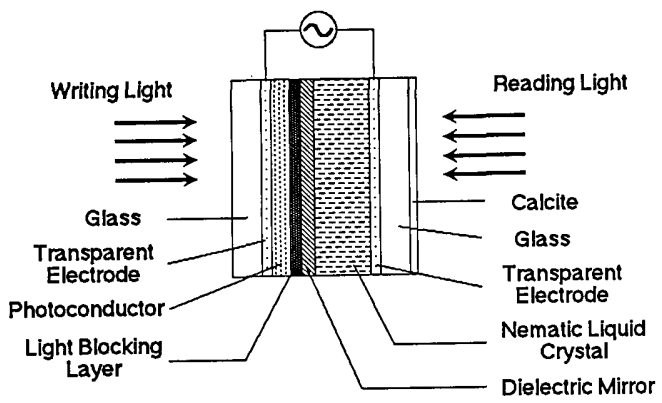

Fig. 10. Considerable encoding device composed of a liquid crystal light valve and a thin calcite plate.

As stated in Sec. III, various types of spatial light modulator can be used as input devices. The device must be capable of converting horizontally polarized light into vertical light and vice versa. In addition, large tolerance for the incident angle of readout light is required because light in each illuminating channel hás to pass the input device at various angles. For example, a liquid crystal light valve attached to a thin calcite plate is considered as such a device (Fig. 10).

Control of the segmented mirror is troublesome. Mechanical movement of mirror elements restricts the operating speed of the system. To increase the operating cycle of the system, more channels than necessary should be prepared and the system should be controlled by switching the channels. In such a system, the necessary sets of operation kernels are loaded and the segmented mirror is adjusted before executing a program; once the program has run, the system is operated by controlling the switching configuration of the source pattern to activate the prestored operation kernels. In this method, mechanical movement of the segmented mirror is an auxiliary control means, so that both high-speed and flexible operation can be expected. The system composed of the birefringent encoder and the multichannel reflective correlator is regarded as a flexible and powerful processor for OAL.

\section{Conclusion}

We have presented a simple image encoding method using birefringence and a flexible correlator with multichannel illumination and a segmented reflector for optical array logic. The system making use of both techniques is constructed and its operation is verified. Necessary devices for the proposed system are a spatial light modulator for a controllable halfwave plate and a controllable segmented deflector.

\section{References}

1. J. W. Goodman, Introduction to Fourier Optics (McGraw-Hill, New York, 1968).

2. A. Huang, "Parallel Algorithms for Optical Digital Computers," in Proceedings, Tenth International Optical Computing Conference (MIT Press, Cambridge, MA, 1983), p. 13.

3. A. W. Lohmann, W. Stork, and G. Stucke, "Optical Perfect Shuffle," Appl. Opt. 25, 1530 (1986).

4. J. Tanida and Y. Ichioka, "Programming of Optical Array Logic. 1: Image Data Processing," Appl. Opt. 27, 2926 (1988).

5. J. Tanida, M. Fukui, and Y. Ichioka, "Programming of Optical Array Logic. 2: Numerical Data Processing Based on Pattern Logic," Appl. Opt. 27, 2931 (1988).

6. H. Fleisher and L. I. Maissel, "An Introduction to Array Logic," IBM J. Res. Dev. 19, 98 (1975).

7. K. Preston, Jr., and M. J. B. Duff, Modern Cellular Automata (Plenum, New York, 1984).

8. M. Born and E. Wolf, Principles of Optics (Pergamon, Oxford, 1975).

9. J. Tanida and Y. Ichioka, "Modular Components for an Optical Array Logic System," Appl. Opt. 26, 3954 (1987).

10. W. Kulcke, K. Kosanke, E. Max, H. Fleisher, and T. J. Harris, "Convergent Beam Digital Light Deflector," in Optical and Electro-Optical Information Processing, J. T. Tippett, D. A. Berkowitz, L. C. Clapp, C. J. Koester, and A. Vanderburgh, Jr., Eds. (MIT Press, Cambridge, MA, 1965), p. 371.

11. A. W. Lohmann and J. Weigelt, "Spatial Filtering Logic Based on Polarization," Appl. Opt. 26, 131 (1987).

12. J. Tanida and Y. Ichioka, "Image Encoding by a Computer Generated Holographic Filter," Proc. Soc. Photo-Opt. Instrum. Eng. 437, 119 (1983).

13. S. H. Lee, Ed., Optical Information Processing Fundamentals (Springer-Verlag, Berlin, 1981).

14. J. Tanida and Y. Ichioka, "Optical Logic Array Processor Using Shadowgrams," J. Opt. Soc. Am. 73, 800 (1983).

15. J. Tanida and Y. Ichioka, "OPALS: Optical Parallel Array Logic System,” Appl. Opt. 25, 1565 (1986). 